\title{
150 Jahre - Ein Rückblick auf das Jahrbuch über die Fortschritte der Mathematik
}

\author{
Silke Göbel, Wolfram Sperber und Bernd Wegner
}

Das Jahrbuch über die Fortschritte der Mathematik (JFM) 1868-1942 ist mehr als ein historischer Zeitzeuge für die

Entwicklung der Mathematik in der zweiten Hälfte des 19. und ersten Hälfte des 20. Jahrhunderts. Als integraler Bestandteil der Datenbank zbMATH bieten die Daten des JFM einen Zugang zur mathematischen Literatur ab der Mitte des 19. Jahrhunderts. Das Jahrbuch hat eine bewegte Geschichte. Es entstand aus einer privaten Initiative, geriet durch den ersten Weltkrieg in eine tiefe Krise, wurde dann von der Preußischen Akademie der Wissenschaften übernommen, die das Jahrbuch noch bis zum Ende des zweiten Weltkriegs unter sich ständig verschlechternden Rahmenbedingungen und zunehmenden politischen Restriktionen weiterbetrieben hat.

Der Artikel beschreibt die Enstehungsgeschichte und die Entwicklung des JFM von 1868 bis heute und würdigt das Wirken der Mathematiker, die federführend daran beteiligt waren. Einen Schwerpunkt bildet die konzeptionelle Diskussion und die Entwicklung der Anforderungen an mathematische Literaturdokumentationsdienste, die heute, im digitalen Zeitalter, vor gänzlich neuen Herausforderungen stehen. Zusätzlich zu den Daten des JFM liegt einiges an Originaldokumenten und Sekundärliteratur in der Berlin-Brandenburgischen Akademie der Wissenschaften (BBAW) vor. Aber nicht alles wurde dokumentiert und vieles ist verschollen, etwa die Daten des JFM für das Jahr 1943.

Wir weisen an mehreren Stellen im Artikel darauf hin.

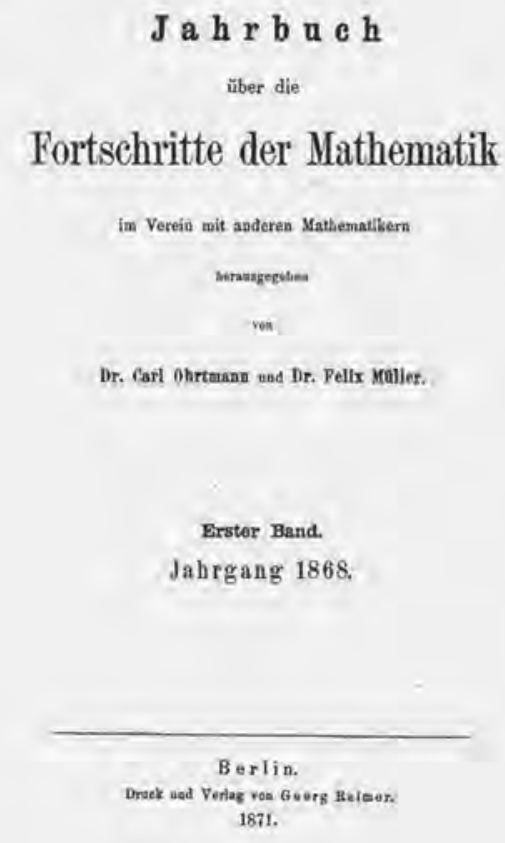

Die Vorgeschichte

Die ersten Veröffentlichungen mathematischen Inhalts in Europa waren mit der Hand geschriebene Bücher; im Mittelalter kamen dann gedruckte Bücher, Flugblätter und Zeitschriften hinzu. Als erste Zeitschriften kennt man die
Roschaer Monatsschrift (1597), Journal des scavans (1665) und Philosophical Transactions of the Royal Society (1665). Im 19. Jahrhundert führte das Anwachsen der wissenschaftlichen Publikationen, insbesondere das Aufkommen wissenschaftlicher Journale, in den Universitäten und Akademien und ihren Bibliotheken zu vielfältigen Initiativen, die Literatur zu sichten, zu ordnen, $\mathrm{zu}$ analysieren und den Wissenschaftlern zugänglich zu machen. Einige Zeitschriften gingen später dazu über, kurze Mitteilungen, Referate genannt, über neu erschienene Bücher oder Arbeiten hinzuzufügen, um den Wissenschaftlern schneller neue Forschungsergebnisse zu vermitteln. Die wachsende Anzahl der Publikationen machte neue Dokumentationsformate, insbesondere wissenschaftliche Referatezeitschriften, erforderlich. 1833 erschien das Jahrbuch für Physiker, Chemiker, Mineralogen, Techniker, Pharmaceuten, Fabrikanten, Oekonomen und alle Freunde der Naturwissenschaften und Technologie, in dem in Form von Aufsätzen neue naturwissenschaftliche Erkenntnise beprochen und vorgestellt wurden. Das Referateorgan der Fortschritte der Physik von 1847 bis 1919 listete die physikalischen Publikationen eines Jahres in systematischer Form (nahezu) vollständig auf. Dieses diente dann als Vorlage für das hier vorgestellte JFM.

Wie es begann - Die Gründung des JFM durch Carl Ohrtmann und Felix Müller

Der Mathematiklehrer Carl Ohrtmann schlug seinem Freund und Kollegen Felix Müller vor, einen Dokumentationsdienst für Mathematik, ähnlich den Fortschritten der Physik, zu gründen. Dieses Jahrbuch sollte die wichtigs- 
ten bibliographischen Angaben über neu erschienene mathematische Veröffentlichungen eines Jahres und kurze Berichte anderer Mathematiker darüber enthalten. Felix Müller war sofort begeistert von Ohrtmanns Idee, die Entwicklung der Mathematik in einem eigenständigen Journal zu dokumentieren, und sagte seine Mitarbeit zur Gründung eines Jahrbuches zu. Ohrtmann und Müller trafen sich daraufhin einige Male, um die Literatur des Jahres $1868 \mathrm{zu}$ sichten und $\mathrm{zu}$ sortieren. Insbesondere die Königliche Bibliothek sowie die Bibliothek der Gewerbeakademie wurden von ihnen anfangs genutzt, um Zeitschriftenverlage in Berlin und potentielle Referenten in Deutschland anzuschreiben. Bei der Adressbeschaffung wurden sie von führenden Berliner Mathematikern, insbesondere Carl Wilhelm Borchardt, Karl Weierstraß und Leopold Kronecker, unterstützt. Diese vermittelten zudem Georg Reimer als Verleger des JFM, der mit den Besonderheiten mathematischer Texte, insbesondere dem mathematischen Formelsatz, vertraut war.

Ohrtmann und Müller, beide promoviert, waren als Oberlehrer im Schuldienst am Königlichen Realgymnasium (später Friedrich-Wilhelms-Gymnasium) in Berlin tätig. Für die Absolventen eines Studiums der Mathematik gab es zu der Zeit noch nicht viele Berufsmöglichkeiten. Versicherungen oder einige große Industriefirmen benötigten Mathematiker mit Kenntnissen in Angewandter Mathematik. An den Universitäten waren Professoren- oder Dozentenstellen für das Fach Mathematik rar. So zogen die meisten, zum Teil auch außergewöhnlich begabte Mathematiker, zunächst die sichere Beamtenlaufbahn an Schulen vor. Viele Lehrer, insbesondere auch Ohrtmann und Müller, waren jedoch weiterhin an aktuellen Forschungsergebnissen auf dem Gebiet der Mathematik interessiert.

Wann die Idee des JFM tatsächlich Gestalt annahm, lässt sich aus den Unterlagen nicht mit Sicherheit rekonstruieren. In einem Vortrag des späteren JFM-Herausgebers Emil Lampe [5] wird das Jahr 1868 als Anfangszeitpunkt benannt. Allerdings verweist Felix Müller in seinem Artikel „Das Jahrbuch über die Fortschritte der Mathematik. 1869-1904“ [8], auf den Dezember 1869 als Geburtsstunde des Jahrbuchs. Der von Müller benannte Zeitpunkt, die Weihnachtsferien im Dezember 1869, erscheint angesichts des heutigen üblichen Vorlaufzeiten der Referateorgane eher unrealistisch, zumal Müller auch auf die Zeitverzögerungen des Erscheinens infolge des deutsch-französischen Krieges hinweist. Andererseits ist ein Brief von Müller an seinen ehemaligen Lehrer Leo Königsberger ein Indiz für 1869. In dem Brief vom 16.3.1870 bittet Müller seinen ehemaligen Lehrer um Unterstützung für das Jahrbuch-Projekt. In der Vorrede steht zu Beginn: „Trotzdem jedoch die Arbeit zu Anfang des vorigen Jahres begonnen wurde, war es nicht möglich, das Erscheinen früher zu bewerkstelligen." Es spricht also einiges dafür, dass die Geburtsstunde des Jahrbuchs auf das Jahr 1868 datiert werden sollte.

Der erste Band des Jahrbuchs mit 838 Einträgen erschien im Februar 1871 . Er enthielt die bibliographischen Angaben und Referate über Veröffentlichungen aus dem Jahr 1868. Dies umfasste 24 deutsche, 18 französische, 12 englische, 13 italienische sowie 12 mathematische Zeitschriften aus anderen Ländern. Die Herausgeber umris- sen ihre Vorstellungen vom Jahrbuch folgendermaßen, siehe [7]:

Das Ziel, das uns vorschwebte, war einerseits: Demjenigen, der nicht in der Lage ist, alle auf dem umfangreichen Gebiete der Mathematik vorkommenden Erscheinungen selbstständig zu verfolgen, ein Mittel zu geben, sich wenigstens einen allgemeinen Überblick über das Fortschreiten der Wissenschaft zu verschaffen; anderseits: dem gelehrten Forscher seine Arbeit bei Auffindung des bereits Bekannten zu erleichtern.

Das setzt Vollständigkeit der Erfassung relevanter Publikationen, objektive Berichterstattung, systematische Einordnung und Aktualität voraus. Nahezu Vollständigkeit wurde zunächst vor allem für die mathematischen Publikationen aus Deutschland angestrebt. Das Referieren der Arbeiten durch andere Mathematiker sicherte die Objektivität der Berichte. Die systematische Zuordnung der Arbeiten wurde durch die Kapiteleinteilung des Jahrbuchs, für die die Herausgeber verantwortlich zeichneten, gewährleistet. Ziel der Herausgeber war es, zeitnah die mathematischen Publikationen eines Jahrgangs zu dokumentieren und inhaltlich zu erschließen. Aktualität war für die Herausgeber ebenfalls ein wichtiges Kriterium für die Relevanz des Jahrbuchs. Alle diese Aspekte sind auch heute noch für die mathematischen bibliografischen Datenbanken wichtig. Im Laufe der Zeit sind neue Kriterien hinzugekommen, und die Relationen zwischen den Kriterien haben sich verschoben. Die konzeptionellen Aspekte mathematischer Literaturdokumentationsdienste werden in einem eigenen Abschnitt diskutiert. Das JFM stellt eine Pionierleistung für den Umgang mit mathematischen Forschungsergebnissen dar. Heute würde man vom Jahrbuch als einem ersten Werkzeug des „Mathematical Knowledge Management" sprechen.

Ihren eigenen Beitrag und ihre Rolle wollten die Herausgeber nicht überbewertet wissen, siehe [7]:

Wir übergeben hiermit dem gelehrten Publikum das erste Heft unseres Jahrbuches. Nicht weil wir uns für die Fähigsten zur Herausgabe desselben hielten, sondern weil wir selbst häufig den Mangel eines solchen empfunden, haben wir die Bearbeitung unternommen.

Ohrtmann leistetet viele Jahre den Hauptbeitrag zum Jahrbuch. Er war für die ersten 15 Jahrgänge verantwortlich, der 16. stand vor der Drucklegung. Er opferte alle Zeit, die ihm neben seiner beruflichen Tätigkeit an der Königlichen Realschule blieb. Als Hobby leistete er sich nur Spaziergänge frühmorgens im Tiergarten und an den Wochenenden in Berlins Umgebung. Auch als sich die Vorboten einer schweren Erkrankung einstellten, arbeitete er mit aller Kraft am Jahrbuch weiter. Als er 1885 nach längerem Krankheitslager starb, würdigte der Oberlehrer Ernst Loew (Biologe) in seiner „Gedächtnisrede“ [6] Ohrtmanns Fähigkeiten, Pflichtbewusstsein und Durchhaltevermögen:

Der letzte Wunsch seines Lebens war, einen Blick auf sein stilles einsames Arbeitszimmer zu werfen. Der 

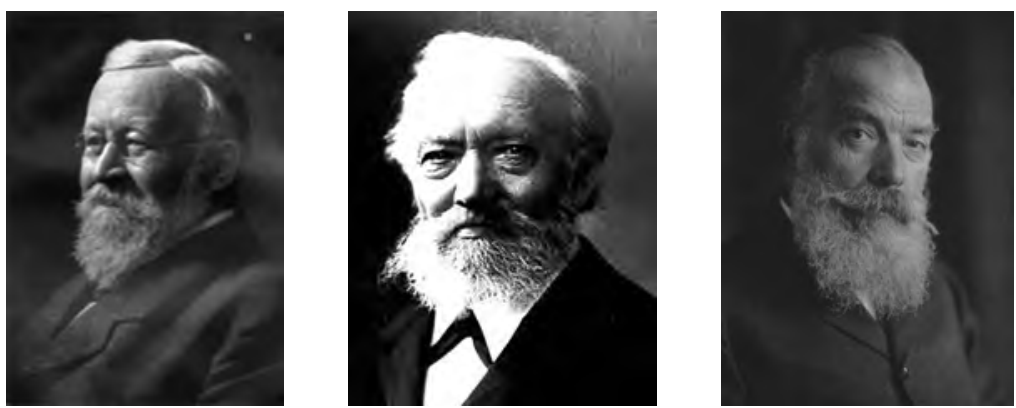

Felix Müller, Albert Wangerin und Emil Lampe (v. 1.n. r.)

Raum, in welchem er sein inneres Sein und Erkennen immer klarer ausgestaltet hatte, dieser bildete sich zuletzt in seinem Auge ab. Dann sank er bewußtlos hin und entschlummerte sanft.

Die Gedächtnisrede bringt dem Leser nicht nur Ohrtmanns Persönlichkeit nahe, sondern verdeutlicht auch die damaligen Ansichten über Pflicht und Bedeutung von Forschung und Wissenschaft.

Der zweite der Gründungsväter des JFM, Felix Müller, beschäftigte sich neben dem Tagesgeschäft auch theoretisch mit verschiedenen Aspekten mathematischer Dokumentationsdienste. Von ihm erschienen neben der bereits zitierten Arbeit weitere allgemeine Publikationen zu diesem Thema: „Über die Bedeutung der Zeitschriften für die mathematische Literatur und die mathematischhistorische Forschung“ [9], „Zur Frage über die Abkürzungen der Titel mathematischer Zeitschriften“ [10], „Versuch einer Gruppierung der neueren mathematisch-historischen Schriften" [11].

Felix Müller war seit 1882 Oberlehrer und wurde 1887 Professor am Luisengymnasium in Berlin. Er wurde 1897 emeritiert und lebte dann als Privatmann in Oberloschwitz bei Dresden und später auf dem Weißen Hirsch bei Dresden. Er war Mitglied der Leopoldina.

$\mathrm{Zu}$ den beiden Gründern des Jahrbuches gesellte sich 1870 Albert Wangerin als weiterer Mitarbeiter.

Wangerin war zu diesem Zeitpunkt Lehrer am Sophiengymnasium in Berlin. 1876 wurde er a. o. Professor an der Universität Berlin und 1882 als Professor an die Universität Halle berufen. Wangerin blieb weiterhin Förderer und Mitherausgeber des Jahrbuches bis 1915. Er verfasste in diesen Jahren 2008 Referate.

In der Regel kamen die Herausgeber wöchentlich zu einer mehrstündigen Sitzung zusammen, um die Arbeitsverteilung $\mathrm{zu}$ besprechen.

Unverzichtbar war die Mitarbeit von Ohrtmanns Frau Luise, die über den Zeitraum von 15 Jahren die Sekretariatsaufgaben wie etwa das Verschicken der Anfragen und Artikel übernahm. Über Frau Ohrtmann ist wenig bekannt. Wahrscheinlich führte sie ihre Tätigkeit ohne jegliche Bezahlung aus. Da die Familie Ohrtmann keine Kinder hatte, nannte Luise Ohrtmann das Jahrbuch häufig „ihr Kind“.

Die Arbeit am JFM erfolgte zum größten Teil auf freiwilliger Basis. Nur den Herausgebern („Schriftleitern“) wurde vom Verlag ein Honorar gewährt. In der Staatsbibliothek Berlin findet man einen Brief vom 25. Juni 1872 von Ohrtmann an Reimer, in dem er auf eine Summe von 100 Talern verzichten würde, wenn „pro Woche 2 Bogen fertig gedruckt werden“. So sehr lag ihm am Gelingen dieses Projektes.

Die Erstellung neuer Werkzeuge zum besseren Zugang zur mathematischen Literatur entsprach dem allgemeinen Zeitgeist. In Deutschland gaben Leo Königsberger und Gustav Zeuner aus Unzufriedenheit mit dem vom JFM verfolgten Konzept der Fremdreferate einen eigenen bibliographischen Dokumentationsdienst heraus, das Repertorium der literarischen Arbeiten aus dem Gebiete der reinen und angewandten Mathematik. Dieser erschien allerdings nur in zwei Bänden. Sie enthielten von den Autoren verfasste Zusammenfassungen ihrer Forschungsergebnisse und beschränkten sich auf wenige Arbeiten. In anderen europäischen Ländern wurden ebenfalls Dokumentationsdienste für die mathematische Literatur aufgebaut, insbesondere in Frankreich, einem wichtigen Zentrum mathematischer Forschung in der zweiten Hälfte des 19. Jahrhunderts. Seit 1870 erschien das Bulletin des sciences mathématiques et astronomique [35] unter der Leitung von Gaston Darboux, das ursprünglich Referate, Buchbesprechungen und Originalartikel enthielt, aber später nur noch Originalartikel publizierte. Unter der Leitung von Henry Poincaré erschien ab 1894 das Répertoire bibliographique des sciences mathématiques [42]. 1912 stellt es sein Erscheinen ein. Hinzu kamen die von Baldassare Boncompagni 1868-1887 herausgegebenen Bände des Bullettino di bibliografia e di storia delle scienze matematiche e fisiche [34] in Italien. Eine Ausnahmestellung nahm der Dokumentationsdienst Revue semestrielle des publications mathématiques [43] (1896-1934) aus den Niederlanden ein, der mit „Berufsreferenten“ arbeitete und einen ähnlichen Ansatz wie das Jahrbuch verfolgte [31].

\section{Die Entwicklung des JFM bis 1930}

Diese Ära wird durch die Mathematiker Lampe, Korn und Lichtenstein geprägt. Schon in den ersten Jahren gab es eine Verzögerung der Herausgabe durch den deutschfranzösischen Krieg 1870/71. Diese führte dazu, dass der zweite Band des JFM erst 1873 erschien. Er enthielt 1629 Einträge aus den beiden Jahren 1869 und 1870 , die in einem Band zusammengefasst wurden. 


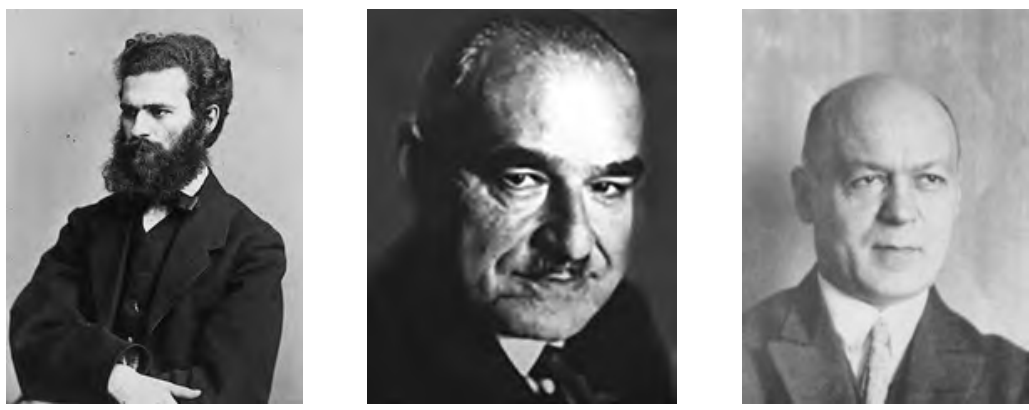

Max Henoch, Arthur Korn, Leon Lichtenstein (v. 1. n. r.)

Nach dem Tod von Ohrtmann übernahm Emil Lampe 1884 einen großen Teil von dessen Arbeit. Er hatte bereits jahrelang als Helfer von Leopold Kronecker beim Journal für Mathematik Erfahrungen in der Herausgabe einer Zeitschrift gesammelt. Von 1866 bis 1889 war er Oberlehrer an der Friedrich-Werderschen Gewerbeschule in Berlin. Zusätzlich lehrte er von 1874 bis 1911 an der Kriegsakademie. 1877 wurde er als Professor an die Technische Hochschule in Charlottenburg berufen. Im Nachruf von Arthur Korn [2] wurde die Referententätigkeit von Emil Lampe als zentraler Bestandteil seiner wissenschaftlichen Tätigkeit herausgestellt. Das äußert sich insbesondere in der immensen Anzahl seiner Referate über nahezu alle mathematischen Gebiete: 16181 , die größte Anzahl von Referaten eines Referenten im Jahrbuch. Außerdem veröffentlichte er 160 wissenschaftliche Arbeiten und zwei allgemeine Artikel zu theoretischen Aspekten der Behandlung mathematischer Literatur: „Ueber die Herstellung eines allgemeinen bibliographischen Repertoriums “[4], „Rückblick und Ausblick“ [5]. Emil Lampe war auch anderweitig für die Verbreitung und Bekanntmachung mathematischer Erkenntnisse aktiv: er war Mitinitiator der Gründung des Mathematischen Vereins an der Universität Berlin und später der Berliner Mathematischen Gesellschaft, die 1901 gegründet worden ist.

$\mathrm{Ab} 1883$ arbeitete Max Henoch, ein Freund Emil Lampes, an der Herausgabe des JFM mit. Henoch war herzkrank, konnte jedoch studieren und sein Studium mit der Promotion bei Karl Weierstraß und Ernst Kummer abschließen.

Eine berufliche Tätigkeit war aber zu anstrengend für ihn. So wurde er Mitarbeiter des Jahrbuchs. Als er 1890 starb, gründete sein Vater die Max-Henoch-Stiftung mit einer Kapitalausstattung von 20 ooo Reichsmark, um die Herausgabe des JFM finanziell zu unterstützen. In der Stiftungsurkunde [37] heißt es:

Auf seinen Wunsch stellte sein Vater beim Tode des geliebten Sohnes der Redaktion die Mittel zur Verfügung, einen Gehülfen für die rein mechanischen äußeren Arbeiten, die - wie wir gesehen - in den ersten 15 Jahren von Frau Ohrtmann ausgeführt wurden, zu gewinnen.

Bei den Stiftungsgeldern sollten möglichst ausschließlich die Zinsen ausgegeben, nur im Ausnahmefall das Kapital angegriffen werden. Sollte die Herausgabe des JFM später einmal eingestellt werden, war das Geld für andere mit der Mathematik zusammenhängende Zwecke bestimmt, deren Verwendung die Physikalisch-Mathematische Klasse der Preußischen Akademie der Wissenschaften festlegen sollte. Das Stiftungsvermögen war aber erst 1920 verfügbar und ging wohl in der Inflation verloren. Unterstützt wurde das Jahrbuch auch von der Berliner Mathematischen Gesellschaft. Detaillierte Hinweise oder schriftliche Unterlagen dazu existieren aber nicht mehr. Es ist nicht klar, in welcher Form die Unterstützung gewährt wurde: finanziell oder durch das Anwerben von Referenten.

Mit Beginn des Jahres 1898 trat Georg Wallenberg als Mitherausgeber der Jahrbuch-Redaktion bei. Er studierte vom WS $1881 / 82$ bis zum WS $1882 / 83$ in Heidelberg und von 1883 bis 1885 in Berlin bei Lazarus Fuchs. Die Promotion erfolgte 1890 in Halle an der Saale. Er war Studienrat und von 1906 bis 1924 a. o. Professor an der Technischen Hochschule Berlin-Charlottenburg. Erich Salkowski, der ab 1906 Mitherausgeber wurde, war zunächst Oberlehrer am KaiserWilhelm-Realgymnasium, dann Privat-Dozent und Professor an der Technischen Hochschule Berlin-Charlottenburg.

Nach dem Tod von Emil Lampe übernahm Arthur Korn 1918 kommisarisch für ein Jahr die Herausgabe des JFM.

Ihm folgten Leon Lichtenstein („eine unvergleichbare Arbeitskraft", wie sich Ludwig Bieberbach später ausdrückte) und ein größeres Team von Mathematikern: Hans Hahn, Erich Hecke, Gustav Herglotz, Otto Hölder, Alfred Loewy, Ludwig Neder, Oskar Perron, George Polya, Gabor Szegö, Hermann Weyl. Die breitere personelle Aufstellung trug der Tatsache Rechnung, dass das ursprüngliche Ziel des JFM, die Entwicklung der Mathematik zu dokumentieren, zunehmend mehr Kapazitäten erforderte.

Der erste Weltkrieg hatte auf das Erscheinen das Jahrbuchs gravierende Auswirkungen: die Literaturbeschaffung wurde immer schwieriger, die Kontakte zu ausländischen Referenten brachen teilweise ab. Das führte zu jahrelangen Verzögerungen bei der Herausgabe des Jahrbuchs. Andererseits betonten führende Mathematiker aus aller Welt wiederholt, dass die Literaturinformationsdienste wichtiger Bestandteil der mathematischen Kultur sind. So stellt Ludwig Bieberbach in seiner richtungsweisenden Rede vor der Akademie 1930 das Jahrbuch in eine Reihe mit Kleins Erlanger Programm. Das gemeinsame Band beider Aktivitäten bestand für Bieberbach im Streben nach „System und Synthese" (siehe [30]) des mathematischen Wissens. 

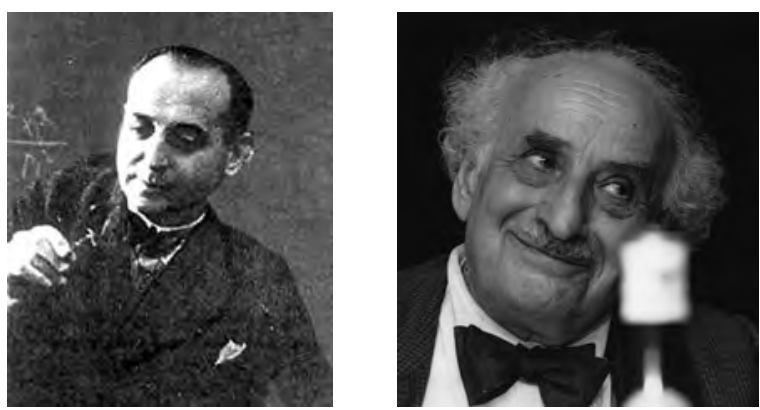

Georg Feigl, Hans Freudenthal (v. 1.n.r.)

Nach Beendigung des ersten Weltkrieges gerieten die deutschen Literaturinformationsdienste in den Fokus der deutschen Wissenschaftspolitik. Den mathematisch-naturwissenschaftlichen Dokumentationsdiensten wurde eine zentrale Rolle zugemessen, um die starke Position Deutschlands in der Mathematik und den Naturwissenschaften vor Beginn des ersten Weltkriegs wieder zu erreichen. Zur Unterstützung und Koordinierung dieser Aktivitäten wurde 1920 die Reichszentrale für naturwissenschaftliche Berichterstattung gegründet. Die Initiative zur Gründung der Reichszentrale ging dabei von der Preußischen Akademie der Wissenschaften aus, die dann im weiteren eine wichtige Rolle bei der Reorganisation der mathematisch-naturwissenschaftlichen Literaturinformation spielte. Insbesondere Max Planck in seiner Funktion als Sekretär der PhysikalischMathematischen Klasse der Preußischen Akademie der Wissenschaften setzte sich immer wieder für die finanzielle Unterstützung des JFM ein [38], was dann ab 1919 von der Preußischen Akademie der Wissenschaften und ab 1920 von der Reichszentrale für naturwissenschaftliche Berichterstattung erfolgte. Hinzu kamen unregelmäßige Zuwendungen von der Notgemeinschaft der Deutschen Wissenschaften, die die Herausgeber nach ihrem Gutdünken verwenden konnten. Also konnten Mitarbeiter des Jahrbuchs auch für Verwaltungsarbeiten bezahlt werden und mehr internationale Literatur beschafft werden. Beispielsweise arbeitete die später bekannt gewordene Hilda Pollazek-Geiringer, verheiratete Geiringer von Mises, als Assistentin von Leon Lichtenstein in der Redaktion des JFM und verfasste 13 Referate. Ob weitere Mathematiker, etwa Herman (Chaim) Müntz, der von 1918 bis 1925 die beachtliche Zahl von 791 Referaten für das JFM lieferte, offiziell als Jahrbuch-Mitarbeiter angestellt waren, ist nicht bekannt.

Bis weit in die zwanziger Jahre hinein hatte das JFM die Zeitverzögerung, die durch den 1. Weltkrieg entstanden war, nicht aufholen können. Erhard Schmidt, Issai Schur und Ludwig Bieberbach, alle drei zur damaligen Zeit Professoren der Friedrich-Wilhelms-Universität, wandten sich deshalb 1927 an die Physikalisch-Mathematische Klasse der Preußischen Akademie der Wissenschaften mit der Bitte auf Übernahme des JFM als akademisches Unternehmen [39]. Deren Vorstandsvorsitzender Max Planck sagte seine Unterstützung zu und legte der Akademie am 23. Juni 1927 einen Vorschlag zur Bildung einer Kommision für das JFM unter seiner Leitung vor [40]. Am 31. Januar 1928 wurde ein
Vertrag zwischen der Akademie und dem Verlag de Gruyter abgeschlossen. De Gruyter war seit 1923 Rechtsnachfolger des Verlages Georg Reimer. Dort wurde auch detailliert vereinbart, welche Partei wie viel Geld zur Verfügung stellen sollte. Der Verlag hatte 5000 Reichsmark pro Band beizutragen, dazu eine Prämie für die fristgemäße Fertigstellung der Bände. Der Schriftleiter sollte von der Akademie bestimmt werden und nur dieser gegenüber verantwortlich sein.

Durch die finanzielle Unterstützung der Preußischen Akademie der Wissenschaften entspannte sich die personelle Situation beim Jahrbuch. Zum 1. Januar 1928 wurde Georg Feigl als hauptamtlicher Schriftleiter bestellt. 1930 wurde Hans Freudenthal Mitarbeiter des Jahrbuchs. Allerdings wechselte Freudenthal noch im selben Jahr an an die Universität Amsterdam und wurde Assistent von L. E. J. Brouwer. Er blieb aber bis 1939 dem JFM insofern treu, als er weiterhin viele Referate schrieb (insgesamt 2323). Nachfolger von Hans Freudenthal wurde der spätere Schriftleiter Helmut Grunsky. Die bessere finanzielle Ausstattung des Jahrbuchs spiegelte sich auch in einer verbesserten Vollständigkeit wider. Band 57 (1931) umfasste 7257 Referate.

Am 9. Mai 1929 gab Max Planck einen Jahresbericht über den Fortgang der Arbeiten in den „Berichten über die wissenschaftlichen Unternehmungen der Akademie" [41] $a b$, für die das Jahrbuch eine ihrer wichtigsten wissenschaftlichen Aktivitäten war.

Allein durch die Nominierung des Schriftleiters hatte die Akademie entscheidenden Einfluß auf die inhaltlichen Zielsetzungen und die redaktionelle Arbeit.

Die Rede von Ludwig Bieberbach, siehe [30], der am 5.11.1931 den Vorsitz der Jahrbuch-Kommision der Akademie von Max Planck übernahm, markiert einen tiefen Einschnitt. In seiner Rede umriss Bieberbach die inhaltlichen Ziele und definiert die Prioritäten für der Weiterführung des Jahrbuchs. Bieberbach unterstrich in seinem Vortrag die Bedeutung des Jahrbuchs und hob dessen Ausnahmestellung hervor. Er hielt dabei aber im wesentlichen an der ursprünglichen Erscheinungsweise des Jahrbuchs fest, das aber zu dieser Zeit sowohl in Deutschland als auch international schon unter heftiger Kritik stand. Im Zentrum der Kritik standen die mangelnde Aktualität und geringe Internationalität des Jahrbuchs. Bieberbach erkannte die Kritik nur teilweise an. Er blockierte damit eine Neuausrichtung und war damit wesentlich verantwortlich für die schwindende Akzeptanz des Jahrbuchs. 


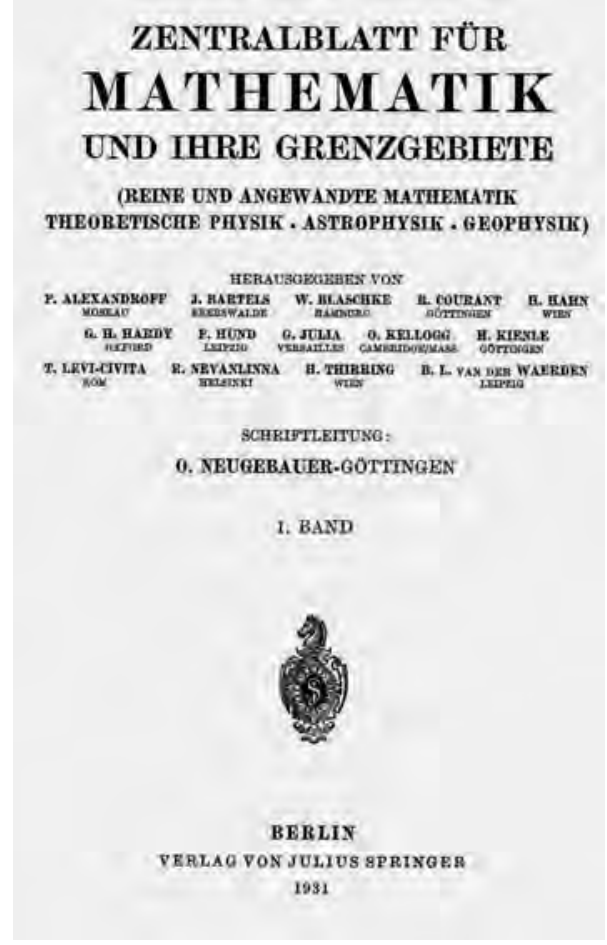

Die Gründung des Zentralblatts, die Entwicklung bis 1945 und die Einstellung des Jahrbuchs

Nach dem ersten Weltkrieg setzte eine internationale Diskussion über die bibliographischen Dokumentationsdienste ein. Vor allem die amerikanischen Mathematiker sahen in der Vorherrschaft deutschsprachiger Dienste eine Benachteiligung und suchten nach Alternativen. Unter anderem wurde über die Gründung eines eigenen Dokumentationsdienstes diskutiert, was aber zunächst verworfen wurde. Ende der zwanziger Jahre verdichteten sich die Anzeichen, dass der Springer-Verlag einen eigenen mathematischen Dokumentationsdienst plant. Der Springer-Verlag hatte die Absicht, die Reihe seiner Dokumentationsdienste, die vom Verlag seit Anfang des Jahrhunderts mit Schwerpunkt Medizin herausgegeben wurden und unter dem Namen Zentralblatt firmierten, zu erweitern. Die Vorbereitungen liefen unter strenger Geheimhaltung und wurden der JahrbuchRedaktion erst im Sommer 1930 bekannt. Als Reaktion auf die Aktivitäten des Springer-Verlags wurden daraufhin Erika Pannwitz (2091 Referate, 1930-1940) und Hans Pietsch (85o Referate, 1930-39) eingestellt. Durch diese Personalverstärkungen konnte der zeitliche Rückstand bei der Herausgabe des Jahrbuchs zwar deutlich aufgeholt, aber nicht beseitigt werden.

Für die Herausgeber des Jahrbuches hatten die Grundsätze „Vollständigkeit“ und „systematische Darstellung" aller Artikel bezogen auf einen Jahrgang Priorität. Das führte aber im Laufe der Jahre dazu, dass mit dem Anwachsen der mathematischen Literatur die Bearbeitungszeit immer länger wurde. Der Springer-Verlag und Mathematiker aus Göttingen, namentlich Otto E. Neugebauer und Richard Courant, ergriffen deshalb nach längeren Diskussionen und
Korrespondenzen die Initiative und gründeten 1931 als Alternative zum JFM das Zentralblatt für Mathematik und ihre Grenzgebiete (ZfM).

Das ZfM setzte seine Prioritäten auf Aktualität und Internationalität, es erschienen mehrere Ausgaben pro Jahr. Es waren auch Englisch, Französisch und Italienisch als Sprachen für die Referate zugelassen.

Die deutschen Mathematiker, insbesondere die Deutsche Mathematiker-Vereinigung, waren angesichts dieser Entwicklung gespalten. Der Konflikt zwischen den Referatejournalen war auch ein Ausdruck der Auseinandersetzung zwischen verschiedenen mathematischen Konzepten und Schulen, insbesondere der mehr international ausgerichteten Göttinger und der eher national orientierten Berliner Schule. Eine Zusammenarbeit oder sogar Einigung auf eine gemeinsame Zeitschrift kam in den Folgejahren nicht zustande. JFM und ZfM strebten beide eine Zusammenarbeit mit der holländischen Referate-Zeitschrift Revue semestrielle des publications mathématiques an. Diese entschied sich - nicht zuletzt durch die Einflussnahme von Hans Freudenthal - für eine Zusammenarbeit mit dem JFM. Konkretes Resultat war der im Jahr 1934 erschienene JFM-Band 59/I, der gemeinsam mit der Revue semestrielle des publications mathématiques herausgegeben wurde und in dem Jan Arnoldus Schouten als Mitherausgeber fungierte.

Der Vertrag wurde aber von der Revue semestrielle des publications mathématiques aus finanziellen und politischen Gründen 1933 gekündigt und die Kooperation eingestellt. Genaueres findet sich dazu in [31].

1935 wurde Helmut Grunsky Nachfolger von Georg Feigl. Weitere Mitarbeiter, die für unterschiedlich lange Zeiträume Arbeiten für das Jahrbuch erledigten, kann man aus den Akten des Archivs der Akademie der Wissenschaften ersehen. Leider waren aber keine schriftlichen Arbeitsverträge auffindbar, meist wurden nur die ausgezahlten Gehälter angegeben. Einige Wissenschaftler aus dieser Liste überbrückten wohl nur kurzzeitig finanzielle Engpässe in ihrer Zeit als Studenten, Doktoranden oder Staatsexamenskandidaten. In den Akten werden folgende Mitarbeiter genannt: Lilly Görke (geb. Buchheim; 1931-1932), Dora Koch (1932-33; 1937?) Willi Rinow (1933-1937), Wilhelm Specht (193334), Edmund Scholz (1934-1939), Maximiliam Pinl (193435), Karl Molsen (1935), Hanna Neumann (geb. Caemmerer, 1937), Wolfgang Hahn (1935-1938), Erwin Schultz (193536), Herbert Jehle (1935-36), Helmut Wielandt (1935-1938), Fritz Dueball (1936-1940), Witold Szablewski (1936-37), Alfred Stöhr (1937), Rudolph Kochendörffer (1937), Wolfgang Wichmann (1940), Gerhard Kropp (1940), Wolfgang Gröbner (1940), Wassilij Höffding (1940-1945), Max Zacharias (1940-45), Wolfgang Franz (1941), Elisabeth Schulenberg (1941-1945), Raschko Zaycoff (1942), Günther Pickert (1939-1943), Hermann von Schelling (1939-1941), Arthur Bischof (1940). Die Mehrzahl dieser Hilfskräfte, darunter relativ viele Doktoranden von Erhard Schmidt und Isaac Schur, machten nach dem 2. Weltkrieg eine Karriere als Wissenschaftler. Isaac Schur war dem Jahrbuch noch aus seiner Zeit als Mitglied der Jahrbuch-Kommission der Akademie besonders verbunden. Als er 1938 aus Deutschland nach Palästina flüchten musste, nahm er alle Bände des JFM mit. 

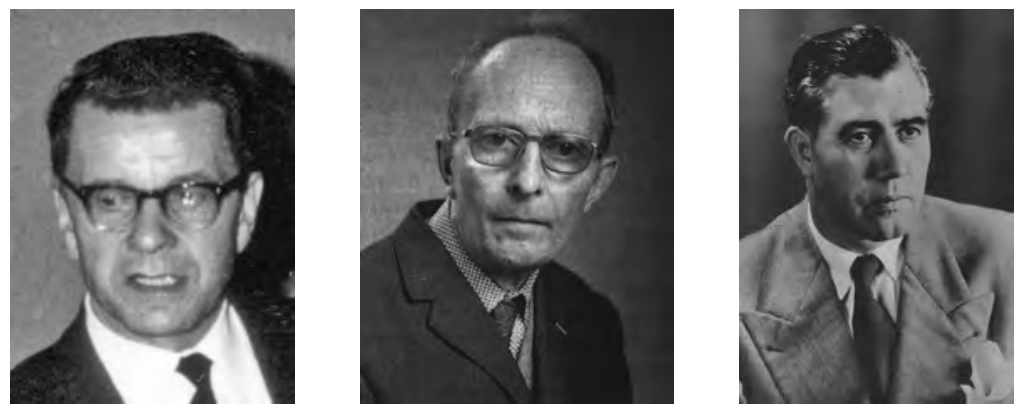

Helmut Grunsky, Christian Pauc, Hermann Ludwig Schmidt (v. 1.n. r.)

Er soll sie abends zum Einschlafen gelesen haben, berichtete Alfred Brauer 1973.

Während der Naziherrschaft versuchten die Redaktionsmitglieder zunächst weiterzuarbeiten wie zuvor. Im Laufe der Jahre wurde Grunsky aber immer mehr unter Druck gesetzt, Juden und „politisch unzuverlässige“ Mitarbeiter $\mathrm{zu}$ entlassen. Ein mahnender Brief von Bieberbach zum Jahreswechsel 1938/39 an Grunsky ist erhalten, in dem Bieberbach beklagt, dass es noch zu viele jüdische Referenten gäbe. Bieberbach war zu dieser Zeit Vorsitzender der mathematisch-naturwissenschaftichen Klasse der Preußischen Akademie der Wissenschaften und Herausgeber des JFM. Nicht lange nach diesem Brief kündigte Grunsky seine Arbeit beim Jahrbuch auf, und der Nationalsozialist Harald Geppert wurde Schriftleiter des Jahrbuchs und „Generalredakteur" der beiden deutschen Referate-Zeitschriften ZfM und JFM. Trotz vieler Verhandlungen, an denen auch die Deutsche Mathematiker-Vereinigung beteiligt war und die in diesem Zusammenhang auch Mitherausgeber des Jahrbuchs wurde, konnte jedoch über eine Zusammenlegung der beiden Dokumentationsdienste keine Einigung erzielt werden. Die Redaktionen arbeiteten weiter unabhängig voneinander, unterstützten sich aber während des Krieges bei der schwieriger werdenden Beschaffung von Zeitschriften und Büchern und übernahmen gegenseitig teilweise auch Referate (888). Es kam auch vor, dass das ZfM ein Referat in Französisch druckte, während man im JFM eine deutsche Übersetzung finden konnte.

Da während des Krieges Wissenschaftler für das Jahrbuch fehlten, versuchte Geppert, französische Kriegsgefangene für die Arbeit in der Redaktion zu gewinnen. Er fuhr 1940 nach Paris und bat den französischen Mathematiker Gaston Julia um Vermittlung. Mehrmals äußerte Julia ausdrücklich als Vorbedingung für seine Unterstützung, dass sein Bruder Roger Julia freigelassen wird. Er nannte Geppert zunächst vier Wissenschaftler, die bereits früher mit deutschen Kollegen zusammengearbeitet haben, und derzeit in Gefangenschaft waren: Jean Leray, Jean Ville, Christian Pauc, Jean Favard.

Während des 2. Weltkrieges wurden französische Offiziere und generell westliche Kriegsgefangene besser behandelt als viele der Gefangenen aus dem Osten. Sie wurden zwar auch in Lagern untergebracht, durften aber für verschiedene Arbeiten die Lager mit Bewachung verlassen und bekamen Lohn. Nach einem aufwändigen Briefwechsel des
Oberkommandos der Wehrmacht mit dem Präsidenten der Preußischen Akademie der Wissenschaften und untergeordneten Angestellten wurden drei Offiziere ausgewählt. Auf einer Besprechung wurde festgesetzt: Es

wurde darauf hingewiesen, daß sich im übrigen die Zusammenarbeit in gesellschaftlichen Formen vollziehen soll, damit die kriegsgefangenen Offiziere nicht das Bewusstsein einer Einengung haben, sondern vollkommen frei wissenschaftlich arbeiten können.

Die ausgesuchten französischen Offiziere waren Professor Dr. Jean Leray, Offizierslager XVII A; Leutnant Professor Christian Pauc, Offizierslager XVII Y; Oberleutnant Professor Dr. Frédéric Roger, Offizierslager XVIII.

Jean Leray lehnte es ab, in Berlin zu arbeiten. Er hatte in dem Lager, in dem er sich befand, eine Universität für die gefangenen Offiziere aufgebaut, die er nicht im Stich lassen wollte. Letztlich blieb es dann nur bei Christian Pauc und Frédéric Roger, die bereit waren, für das Jahrbuch zu arbeiten (Erlass vom 8. November 1942). Sie wurden in das „kleine Stammlager III D Falkensee“ gebracht, und fuhren von dort jeden Morgen (erst mit, später ohne Bewachung) zur Preußischen Akademie der Wissenschaften (Arbeitszeit 9-16 Uhr).

Am 10. Mai 1943 wurden die beiden Franzosen in ein „Zivildienstverhältnis“ überführt und die Akademie wurde angewiesen, für sofortige Unterkunft zu sorgen. Es wurden Verträge geschlossen, dass die beiden eine Vergütung von 350 Reichsmark für die Tätigkeit als wissenschaftliche Hilfsarbeiter beim Jahrbuch bekommen sollten. Der Lohn sollte bis zum 8.5.1945 fest bleiben (welche Ironie des Datums!!).

Am 23. Juni 1943 stellte Geppert einen Antrag an die Preußische Akademie, die Ehefrauen von Pauc und Roger vom 1. Juli bis Ende September 1943 als Volontärinnen (ohne Bezahlung) für das Jahrbuch anzustellen. Dem wurde zugestimmt und deren Vertrag bis zum 30.1.1944 verlängert. Am 1. Dezember 1943 traf Pauc das Schicksal wie viele andere Berliner. Er verlor bei einem Brand seine Wohnung und hat fast alle persönlichen Sachen eingebüßt. Im November 1943 beantragten beide, an einer anderen Universität innerhalb Deutschlands arbeiten zu dürfen. Pauc wurde von Otto Haupt eine planmäßige Assistentenstelle in Erlangen angeboten. Gleichzeitig bekam Frédéric Roger vom Rektor 
der Universität Freiburg, Prof. Dr. Wilhelm Süss, eine Stelle an der Universität Freiburg. Beide haben aber den Wunsch geäußert, weiterhin an der Redaktion des Jahrbuchs freiwillig mitwirken zu können.

Nach dem Krieg 1945 kehrte Pauc wieder nach Frankreich zurück, hatte aber zunächst Probleme eine adäquate Stelle zu finden, da er mit den Deutschen "fraternisiert“ hatte. Von 1948 bis 1952 lehrte er an der Universität Kapstadt, bevor er 1953 nach Nantes wechseln konnte. Seine Zeit in Deutschland sah er aber nicht negativ (siehe dazu [15]). Pauc arbeitete nach dem Krieg noch einige Jahre beim Zentralblatt mit. Vom weiteren Schicksal Frédéric Rogers ist nichts bekannt; nach einer mündlichen Mitteilung von Christophe Eckes überlebte er aber den Krieg.

Was die Auswahl der Artikel für das Jahrbuch und den Inhalt der Referate betrifft, ist bis in den 2. Weltkrieg hinein kaum ein Einfluss der Nationalsozialisten zu erkennen. Arbeiten von jüdischen Autoren, etwa R. Courant, W. Döblin, A. Einstein, F. Hausdorff, H. Rademacher, wurden weiterhin objektiv besprochen. Erika Pannwitz schrieb 1947: „Es gibt keinen Fall, in dem eine Arbeit deshalb kurz besprochen oder ohne Referat geblieben wäre, weil der Verfasser Jude war.“ Auch Hermann Weyl sagte nach dem Krieg: „It is true that even during the war the Jahrbuch continued reviewing the papers of foreign and Jewish mathematicians in an objective and decent manner" [30]. Nur in einigen einzelnen Referaten, vor allem aus dem Abschnitt Pädagogik in den Bänden 59, 6o und 61 sind die politischen Ansichten dieser Zeit zu erkennen. Aus politischen Gründen waren Referate zu Artikeln mit Titeln wie „Der mathematische Unterricht im Dritten Reich“, „Mathematik im Dienste der nationalpolitischen Erziehung“ oder „Verdeutschung mathematischer Fachausdrücke" unvermeidbar. Ab 1936 wurde auf das Kapitel Pädagogik im JFM verzichtet.

Der letzte ausgelieferte Band des JFM war der erste Halbband des Bandes 68 (1942). Im Laufe der Jahre 1945 und 1946 kehrten einige ehemalige Mitarbeiter nach Berlin zurück, die sofort mit dem Ordnen und Aufräumen begannen. Insbesondere Frau Dr. Schulenberg und Frau Henschel sind hier zu nennen. Herrmann Ludwig Schmid, der Direktor des Mathematischen Instituts der Universität Berlin, verfasste am 29. August 1945 ein Memorandum über den Stand der Arbeiten am Jahrbuch. Teile der Jahrbuchbände von 67 bis 71 waren noch aufzufinden. H. L. Schmid regte auch gleich an, zügig mit drei Mitarbeitern weiterzuarbeiten. Er wollte eine so große Lücke, wie es sie nach dem 1. Weltkrieg gegeben hatte, vermeiden. In einem weiteren Brief vom 15.9. 1945 schlug er außerdem Dr. Rudolf Kochendörffer als Mitarbeiter vor. In dieser Zeit war es nicht einfach, geeignete Mitarbeiter zu finden. Stellenbewerber mussten nachweisen, keine Nazis gewesen zu sein. Die Organisation des Jahrbuches lag weiterhin bei einer Kommission des Forschungsinstituts für Mathematik der Deutschen Akademie der Wissenschaften (die Preußische Akademie war 1946 umbenannt worden).

Es musste nun geklärt werden, welches Referateorgan mit welchem Verlag weiter geführt werden sollte. Der Springer-Verlag (West) bestand auf der Fortsetzung seiner Arbeit mit dem Zentralblatt. Der neu gegründete Akademie-
Verlag (Ost) wollte mindestens das Jahrbuch übernehmen, wenn nicht beide Referatorgane zusammen. Gegen die Übernahme des Jahrbuches sprach aber ein alter Vertrag des de Gruyter-Verlages mit dem Jahrbuch. Die Verhandlungen der Akademie mit dem Springer-Verlag verliefen zügig, so dass das Zentralblatt schon am 1.1.1947 wieder erscheinen konnte, während sich die Akademie und der de Gruyter-Verlag nicht einigen konnten.

Am 5.11.1947 schrieb Erhard Schmidt in einem Tätigkeitsbericht des Forschungsinstituts für Mathematik u.a. über die Situation beim Jahrbuch und äußerte die Hoffnung, dass das Jahrbuch vom Jahre 1948 wieder erscheint. Am 10. Februar 1948 wurde Hermann Ludwig Schmidt die Redaktion des Jahrbuchs übertragen. Vorher hatte er einige Male mit dem de Gruyter-Verlag verhandelt, der sich aus technischen Gründen jedoch zu dieser Zeit nicht in der Lage sah, den Druck des Jahrbuches zu übernehmen. Am 7. Mai 1948 kündigte der Direktor der Deutschen Akademie Josef Naas den Vertrag mit dem de Gruyter-Verlag zum Jahresende 1948 und das Jahrbuch wurde eingestellt. Die mathematische Welt reagierte unterschiedlich auf das Ende des Jahrbuchs. Man sah ein, dass einerseits das Erscheinen von drei ähnlichen mathematischen Referateorganen in mancher Hinsicht nicht nötig war (seit 1940 wurden in direkter Konkurrenz zum Zentralblatt die Mathematical Reviews in den USA herausgegeben). Andererseits lobten einige Mathematiker besonders die Gründlichkeit des Jahrbuchs, die Systematik und fanden eine Konkurrenz gerade angebracht.

\section{Zur konzeptionellen Entwicklung des Jahrbuchs}

\section{Vollständigkeit und Aktualität}

Die angestrebte (möglichst) vollständige Erfassung der mathematischen Literatur wurde bereits im Vorwort des ersten Jahrbuchs [7] als ein wesentliches Ziel benannt. Was heißt Vollständigkeit? Bis ins 20. Jahrhundert wurden aktuelle mathematische Forschungsergebnisse in Form von Publikationen der mathematischen Community mitgeteilt. Das Ziel Vollständigkeit wäre dann erreicht, wenn man alle mathematischen Publikationen erfasst und ausgewertet hat. In der Praxis ist die vollständige Erfassung der Mathematikrelevanten Publikationen insbesondere in mathematischen Anwendungsgebieten unrealistisch. Man beschränkte sich daher auf die Auswertung der ausgewiesenen mathematischen Zeitschriftenserien sowie Monographien und Lehrbücher und ausgewählter Zeitschriftenserien aus anderen Wissenschaften. Selbst dieses konnte in den ersten Bänden nur mit Abstrichen erreicht werden. Im ersten Band wurden von (etwa) 350 relevanten Zeitschriften weltweit 78 ausgewertet. Durch die Vorgehensweise, die mathematischen Publikationen jahrgangsweise zusammenzufassen, war die angestrebte Vollständigkeit eine der Hauptursachen für die zeitliche Verzögerung des Erscheinens der JFM-Bände. Auf Vorschlag von Max Planck wurden ab dem Jahrbuch Band 1928 die Publikationen eines Jahres auf zwei Halbbände verteilt. Das führte zu mehr Flexibiltät und einer Verkürzung der Spanne zwischen dem Erscheinen einer Publikation und der Besprechung im Jahrbuch. Damit konnte der Rückstand 
in der Aktualität gegenüber dem Zentralblatt aber nicht ausgeglichen werden. Der vom Zentralblatt verfolgte Ansatz, Arbeiten so schnell wie möglich zu dokumentieren, trug besser zur erforderlichen Aktualität bei. Die mathematischen Forschungskapazitäten wurden in der ersten Hälfte des 20. Jahrhunderts weltweit massiv ausgebaut, und mathematische Methoden und Ergebnisse fanden unmittelbar Eingang in innovative Technologien und Produkte, was der Forderung über schnelle Information zu aktuellen Forschungsergebnissen hohe Priorität verlieh.

\section{Systematik im Jahrbuch: Kapiteleinteilung, Referate, Registerbände}

Ein weiteres zentrales Anliegen des JFM war die systematische inhaltliche Einordnung der Publikationen. Das vom JFM verwendete dreistufige Schema der Kapitelüberschriften, das maßgeblich von den Herausgebern in enger Abstimmung mit fachlichen Experten verantwortet wurde, ermöglichte eine sachbezogene Einordnung der Referate von unterschiedlichem Präzisionsniveau. Die Besprechungen der Arbeiten enthielten auch Zitate von früheren Publikationen, die für die jeweilige Arbeit von Relevanz waren. Zusätzlich wurden Hinweise auf deren Besprechungen im JFM angegeben. Die inhaltliche Vernetzung war ein wichtiges Hilfsmittel, um sich über die Entwicklung eines Sachgebiets oder Teilen davon zu informieren. Allerdings stellten die Kapitelüberschriften im Jahrbuch im engeren Sinn noch kein Klassifikationssystem dar. Es fehlen formale Klassenbezeichner, in denen sich auch die hierarchische Strukturierung der inhaltlichen Aspekte widerspiegelt. Zudem wurden die Kapitelüberschriften häufig modifiziert, was der Entwicklung der Mathematik entsprach, aber eine inhaltliche Suche erschwerte. Eine Gruppierung der Publikationen nach inhaltlichen Kriterien war eine wesentliche Voraussetzung für die nachhaltige Akzeptanz und Bedeutung des JFM. Die systematische Zuordnung der Publikationen zu mathematischen Gebieten war kein Alleinstellungsmerkmal des Jahrbuchs, das Zentralblatt verwendete ein ähnliches Schema.

Die beiden Jahrbuch-Gründer waren sich einig, dass möglichst nur Fremdreferate im Jahrbuch erscheinen sollten. Das erforderte den Aufbau eines Referentenpools. In den ersten Bänden stammten die Referenten vorwiegend aus Deutschland. Aber bereits ab dem zweiten Band engagierten sich auch Mathematiker aus anderen europäischen Ländern. Die Anzahl der „Herren Referenten“ (mit Band 44 (1913), der im Jahre 1918 erschien, wurde der Sprachgebrauch dann in "Mitarbeiter" geändert) stieg pro Band von anfangs 16 bis auf ca. 300 in den dreißiger Jahren.

Viele nahmhafte Mathematiker beteiligten sich als Referenten am Jahrbuch. Aus dem Ausland sind etwa zu nennen: Enea Bortolotti (569, Florenz), Bohumil Bydzovsky (503, Prag), Arthur Cayley (685, Cambridge), Gustav Eneström (796, Stockholm), Hans Freudenthal (2311, Amsterdam), Rudolf Fueter (601, Zürich), James Glaisher (1498, Cambridge), Sophus Lie (96, Christiania und Leipzig), Gino Loria (1230, Genua), Paul Mansion (1004, Gent), Nikolaj Sincov (1310, Leningrad), Gabor Szegö (1850, Stanford), Francesco Tricomi (804, Turin), Gulio Vivanti (1740, Mailand), aus Deutschland: Alfred Clebsch (Göttingen, 5), Da- vid Hilbert (Königsberg und Göttingen, 262), Felix Klein (München, 32), Oskar Perron (München, 756) und Hermann Weyl (Göttingen und Zürich, 199). Hier müssen natürlich auch Emmy Noether (Göttingen, 78 Referate 1915-28) und Dorothy Maud Wrinch (Cambridge, 39 Referate 1914-19), erwähnt werden, die zu den ersten Referentinnen des JFM zählten. Die Referenten wurden im Jahrbuch teilweise abgekürzt sowie mit Titel und Wohnort angegeben.

Die Referententätigkeit war zwar mit einer hohen Wertschätzung verbunden. Die Besprechung der Arbeiten erforderte allerdings einen beträchtlichen Aufwand. Die Referate wurden aber nicht als eigenständige Publikationen angesehen. Viele Mathematiker waren daher zögerlich, als Referenten in einem Dokumentationsdienst mitzuarbeiten, was auch heute noch zutrifft.

Die Referate waren meist kurze, sachliche Einträge über den mathematischen Inhalt. Es gab aber auch lange Berichte, die über mehrere Seiten gingen. Dabei handelte es sich oft um Referate über Bücher oder besonders herausragende Publikationen. Emil Lampe formulierte 1904 Richtlinien zur Anfertigung der Referate [5]:

Das Jahrbuch soll den Leser darüber belehren, was in den Abhandlungen steht, nicht was der Referent über den Inhalt denkt. Zum Zwecke der eigenen Forschung soll der Benutzer des Jahrbuches erfahren, ob ein Aufsatz neue Gedanken enthält ... Nur offenbare Unrichtigkeiten sind in nicht verletzender Form zu bezeichnen.

Die Bewertung fehlerhafter Arbeiten fiel zum Teil aber drastisch aus, wie die folgenden Beispiele zeigen:

- JFM 03.0324.01: „Falscher Schluss aus dem Besondern auf das Allgemeinere ..."

○ JFM 06.0311.01: „Der dritte Abschnitt enthält im Wesentlichen vier neue Beweise; die vier Beweise sind falsch ..."

- JFM 07.0001.01: „Es lohnt nicht der Mühe, alle falschen, längst widerlegten Behauptungen, die das Programm enthält, aufzuführen; jedenfalls muss man vor allzu großer Vertrauensseligkeit den Behauptungen des Verfassers gegenüber ernstlich warnen ..."

- JFM 11.0596.03: „Der Verfasser kennt nichts von der Literatur über den Gegenstand, so dass er von der falschen Voraussetzung ausgeht, die ..."

- JFM 15.0057.03: „Falsch! Herr W. verwechselt notwendige und hinreichende Bedingungen ..."

- JFM 21.0093.03: „Eine Arbeit, in der viel Mühe ganz vergeblich aufgewandt worden ist ..."

- JFM 27.0042.03: „Mangel an wissenschaftlicher Strenge und Gründlichkeit ... Der Verf. lebt in seiner Gedankenwelt und fordert, dass alle übrigen Gelehrten sich ihm anbequemen sollen ..."

- JFM 68.0100.01: „Es ist tief bedauerlich, dass ein derartiges Buch erscheinen durfte ..."

Die von Lampe formulierten Richtlinien machen die Schwierigkeit öffentlicher kritischer Bewertungen in den Referateorganen deutlich. Technische Arbeiten erfordern einen hohen Aufwand, um die Ergebnisse zu verifizieren, und die 

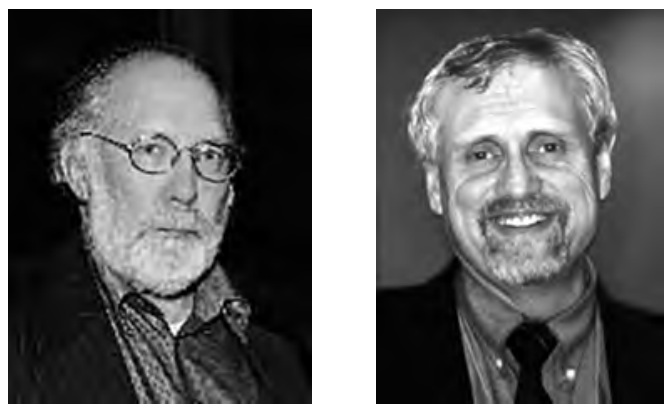

Bernd Wegner, Keith Dennis (v. 1.n.r.)

Tragweite neuer Ansätze ist oft nicht unmittelbar zu erkennen, so dass kritische Referate, wenn sie nicht auf offensichtlichen Fehlern beruhen, der besonderen Aufmerksamkeit der Herausgeber bedürfen. Eine ausgewogene Berichterstattung über die Publikationen ist aber entscheidend für die Reputation des Dienstes. In gewisser Weise hat das JFM mit kritischen Beiträgen auch eine Art öffentlicher Bewertung der mathematischen Literatur bis 1942 übernommen, die über eine Auflistung, Beschreibung und Systematisierung der aktuellen Literatur hinausgeht und von der mathematischen Leserschaft durchaus geschätzt und anerkannt wurde: „The most important aid to judge contemporaneous work is furnished by a German publication known as the Jahrbuch über die Fortschritte der Mathematik“, schrieb 1912 der Amerikaner G. A. Miller [26].

Die Referententätigkeit erfolgte zunächst ohne Entgelt. In späteren Jahren wurde eine Reichsmark als Aufwandsentschädigung für die Anfertigung eines Referates gezahlt.

Des Weiteren enthielt jeder Band auch ein Inhaltsverzeichnis, Verzeichnis der Autoren und Referenzen, sowie ein Zeitschriftenverzeichnis. Das Zeitschriftenverzeichnis war wichtig, da die meisten Zeitschriftennamen abgekürzt wurden.

\section{Internationalität}

Das Jahrbuch war zwar eine deutsche Unternehmung, was durch die herausgehobene Stellung der mathematischen Forschung in Deutschland historisch auch berechtigt war. Von Anfang an wurden aber, wie oben dargelegt, die mathematischen Publikationen weltweit ausgewertet und im Jahrbuch angezeigt. Bis 1930 hatten sich aber die Kräfteverhältnisse in der mathematischen Forschung mit dem breiten Ausbau mathematischer Forschung in den USA, Japan und der Sowjetunion entscheidend verändert. Das JFM übernahm die Titel der Arbeiten zwar in der Originalsprache, die Referate eschienen aber immer auf Deutsch. Das ZfM hingegen akzeptierte auch Referate in anderen Sprachen, was die Sprachbarriere vor allem für die englischsprachigen Mathematiker aufhob. Auch hinsichtlich des Referentenpools unterschieden sich JFM und Zentralblatt deutlich. Der Referentenpool des ZfM war internationaler und jünger als der des JFM.

Zusammenfassend lässt sich feststellen, dass der ursprüngliche Ansatz des Jahrbuchs im Jahr 1930 an seine
Grenzen gekommen war und dass die Reorganisation, insbesondere die Umstellung auf die halbjährliche Erscheinungsweise, unzureichend war. Allerdings sind vom ZfM und den Mathematical Reviews (MR) viele Ansätze des Jahrbuchs in modifizierter Form aufgegriffen, implementiert und erweitert worden, insbesondere die systematische Erfassung,

\section{Das JFM nach 1945}

Die Geschichte des JFM war aber mit der Einstellung der Herausgabe des Jahrbuchs noch nicht zu Ende. Selbst in der Zeit, wo es das ZfM und die MR nur als bibliographische Dokumentationsdienste auf Papier gab, wurde die Einbeziehung der JFM-Referate in die Referate von ZfM und MR fortgesetzt: Zitate, die in die Zeit fielen, in der es nur das JFM gab, wurden durch einen Hinweis auf das entsprechende Referat im JFM ergänzt. Beide Dienste führten entsprechende Hinweise im Impressum auf, die MR längere Zeit mit einem JFM-unfreundlichen Tenor.

Mit dem Übergang von ZfM und MR in das digitale Zeitalter gab es eine Wiederbelebung der alten Datenbestände des JFM. 1997 schlugen die damaligen Chefredakteure des ZfM, Bernd Wegner (TU Berlin), und der Mathematical Reviews, Keith Dennis (Universität Cornell, USA), vor, das JFM zu digitalisieren und in einer Datenbank allgemein zugänglich zu machen. Die Bereitstellung der JFMDaten als Datenbank würde die Suche und das Auffinden der mathematischen Literatur aus der zweiten Hälfte des 19. Jahrhunderts und der ersten Hälfte des zwanzigsten Jahrhunderts wesentlich vereinfachen. Die Digitalisierung des JFM war Teil einer größeren Idee, eine digitale Bibliothek der Mathematik aufzubauen, die später von der International Mathematical Union aufgegriffen und verallgemeinert worden ist, siehe [44]. Mit finanzieller Unterstützung der Deutschen Forschungsgemeinschaft (DFG) wurde 1997 das Projekt „Electronic Research Archive for Mathematics“ (ERAM) gestartet, an dem die TU Berlin, die Staats- und Universitätsbibliothek (SUB) Göttingen und das FIZ Karlsruhe beteiligt waren.

Ziele des Projekts waren einerseits die Erfassung und Aufarbeitung sämtlicher Daten aus dem gedruckten JFM in einer Datenbank, die Digitalisierung wichtiger mathematischer Publikationen und Dokumente und die Vernet- 
zung zwischen JFM Daten und Volltexten. Inzwischen enthält das an der SUB Göttingen aufgebaute digitale Archiv (www.gdz-cms.de) eine Reihe der früheren Jahrgänge renommierter Zeitschriften wie etwa die Mathematische Annalen, die Mathematische Zeitschrift oder die Commentarii Mathematici Helvetici, sowie Nachlässe bedeutender deutscher Mathematiker.

Alle bibliographischen Daten und Referate aus dem gedruckten Jahrbuch wurden von Schreibbüros in Berlin, Prag und Sofia manuell erfasst und in der Redaktion des ZfM in einer Datenbank zusammengeführt. Als Format wurde $\mathrm{T}_{\mathrm{E}} \mathrm{X}$ verwendet, was auch die Voraussetzung für mathematikspezifische Retrievalfunktionalitäten ist, insbesondere bei der Formelsuche. Die digitale Erfassung der JFM-Daten ist inzwischen abgeschlossen. Diese Datenbank ist im Web [36] unter der Herausgeberschaft der European Mathematical Society frei zugänglich. Außerdem wurden alle Daten in die Datenbank zbMATH [45], die Online-Version des ZfM, eingefügt.

2005 wurde das JFM Digitalisierungsprojekt mit dem „2005 PAM Division Award“ von der Special Libraries Association (Physics-Astronomy-Mathematics Division) „for its significant contribution to the field of mathematics" gewürdigt.

Die Aufarbeitung, Vervollständigung und Erweiterung der JFM-Daten war in vielerlei Hinsicht langwierig und aufwändig und ist noch nicht vollständig beendet.

- Die JFM Einträge waren nicht entsprechend dem heute verwendeten Klassifikationsschema MSC klassifiziert, sondern nur in Kapitel sortiert, und enthielten keine Verschlagwortung. Im Projekt sollten die Enträge durch eine Klassifikation gemäß der heute verwendeten MSC und Schlagwörter (in Englisch) ergänzt werden.

- Der Einfluss wichtiger Publikationen auf die moderne Mathematik sollte durch eine Neubewertung der Arbeiten erweitert werden.

- Die Autorennamen im JFM beschränkten sich meistens auf die Nachnamen, Transliterationen der Autorennamen erfolgten nach unterschiedlichen Regeln. Diese Angaben sollten so weit wie möglich vervollständigt und vereinheitlicht werden.

- Die Quellenangaben, insbesondere die Zeitschriftentitel, entsprechen nicht den heutigen Standards und wechselten im Laufe der Zeit. Die Quellenangaben sollten standardisiert werden.

- Die JFM-Referate waren überwiegend in deutscher Sprache, während die Titel in der originalen Sprache und Schreibweise angegeben waren. Hier sollte eine Übersetzung der Titel ins Englische erfolgen.

- Eine Schwierigkeit für das Einfügen der JFM-Daten in eine gemeinsame Datenbank ergab sich aus der unterschiedlichen Struktur der JFM- und ZfM-Daten, so dass eine Transformation der Datenstruktur erforderlich war.

- Die Literaturreferenzen im JFM sollten in HypertextLinks überführt und ergänzt werden. Das umfasst zwei Arten von Links: Interne Links (von JFM-Einträgen auf andere JFM-Einträge) und externe Links (von JFMEinträgen auf die digitalisierten Volltexte).

\section{Special Libraries Association} Physics-Astronomy-Mathematics Division

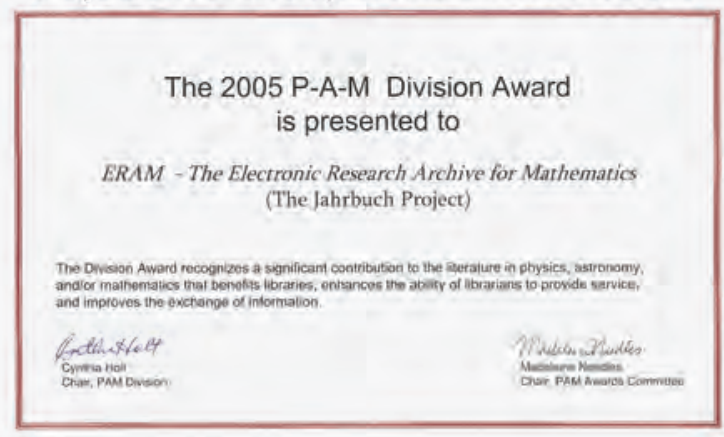

Die Aufarbeitung der JFM-Daten, die auf der freiwilligen Mitarbeit vieler Mathematiker beruhte, erwies sich als aufwändiger als gedacht und wird heute partiell (insbesondere durch Hinzufügen der Links und Erstellung von Autorprofilen) im Rahmen der Weiterentwicklung der Datenbank zbMATH weitergeführt.

Wir möchten uns bei allen Wissenschaftlern (zeitweise haben bis $\mathrm{zu}_{50} \mathrm{Wissenschaftler} \mathrm{am} \mathrm{Projekt} \mathrm{mitgearbeitet)}$ bedanken, die bei der Digitalisierung und Aufbereitung der JFM Daten mitgewirkt haben.

Epilog

Mit dem JFM ist 1869 ein erstes Instrument für mathematisches Wissensmanagement entstanden, das die damaligen Möglichkeiten des Printmediums ausschöpfte. Das JFM entstand aus dem Enthusiasmus einiger Mathematiker, die die Notwendigkeit und den Nutzen eines solchen Dienstes für die mathematische Community sahen. Die Akzeptanz des JFM und dessen Nützlichkeit hat sich nicht zuletzt in dessen Erscheinen bis zum Ende des zweiten Weltkriegs erwiesen. Mit der Digitalisierung des JFM stehen nun weltweit allen Nutzern dessen Daten im Internet zur Verfügung. Die mathematische Literatur stellt bis heute den Kern des mathematischen Wissens dar und ist im wesentlichen heute digital verfügbar, weltweit verteilt auf viele Provider. Die Datenbank zbMATH ist dabei ein effizientes Zugangswerkzeug zur mathematischen Literatur. Mit der Integration der JFM-Daten in zbMATH steht der mathematischen Community ein einzigartiges Werkzeug zur Verfügung, die Entwicklung der Mathematik seit der Mitte der 19. Jahrhunderts bis heute $\mathrm{zu}$ verfolgen.

Heute steht das Referatewesen vor neuen grundlegenden Herausforderungen und Änderungen. Im Internet werden neue Forschungsergebnisse vorab als Preprints oder, in begutachteter Form, in von Verlagen und Gesellschaften herausgegegeben Journalen bereitgestellt. Die großen Internetsuchmaschinen wie Google machen mathematische Publikationen weltweit zeitnah auffindbar, aber es mangelt 
an Vollständigkeit. Auch die sachliche Erschließung und Standardisierung der Daten lässt bei Google zu wünschen übrig. Vor allem in Anwendungsbereichen ist die Anzahl mathematischer Publikationen weiter stark im Wachsen. Darüber hinaus haben sich vielfältige Informationsdienste für die Mathematik etabliert, die Daten über mathematische Objekte wie etwa Gruppen, spezielle Polynome und Ideale oder Zahlenfolgen bereitstellen. Mathematische Software schließlich ist zu einer neuen eigenständigen Säule des mathematischen Wissens und seiner Anwendungen geworden.

Bei der aktuellen Informationsflut, auch bei mathematischen Publikationen, sind eine systematische Erfassung, eine tiefere inhaltliche Erschließung, die über die bibliographischen Daten hinausgeht und bis zur Formelanalyse reicht, und die (semantische) Vernetzung mit relevanten Informationen, seien es Publikationen, Software, mathematische Modelle oder andere Forschungsdaten, neue Herausforderungen für Datenbanken für mathematische Dokumentationsdienste.

\section{Verzeichnis der Quellen}

\section{Arbeiten der Herausgeber des JFM}

[1] Helmut Grunsky: Collected papers. Edited by Oliver Roth and Stephan Ruscheweyh. With an introduction by Christian Pommerenke and a contribution by Reinhard Siegmund-Schultze. Lemgo: Heldermann Verlag, 48o p. (2004) (Zbl1065.01017)

[2] Arthur Korn: Emil Lampe: Jahrbuch über die Fortschritte der Mathematik. Band 45. Berlin: G. Reimer, p. I-VII (1914/1915)

[3] Emil Lampe: Dr. Max Henoch: Jahrbuch über die Fortschritte der Mathematik. Band 20. Berlin: G. Reimer. p. 1-6 (1891)

[4] Emil Lampe: Ueber die Herstellung eines allgemeinen bibliographischen Repertoriums. Deutsche Math. Ver. 4, p. 129-131 (1897) (JFM 28.0070.03)

[5] Emil Lampe: Rückblick und Ausblick. Jahrbuch über die Fortschritte der Mathematik. Band 33. Berlin: G. Reimer. p. 1-5 (1904)

[6] Ernst Loew: Gedächtnisrede für Karl Ohrtmann. Jahresbericht/Königliche Realschule zu Berlin. p. 27-34 (1885/86)

[7] Carl Ohrtmann, Felix Müller, Vorrede. Jahrbuch über die Fortschritte der Mathematik. Band 1. Berlin: G. Reimer. p. I-IV (1871)

[8] Felix Müller: Das Jahrbuch über die Fortschritte der Mathematik. 1869-1904. Bibl. Math. 5 (3), p. 292-297 (1904) (JFM 35.0042.03)

[9] Felix Müller: Über die Bedeutung der Zeitschriften für die mathematische Literatur und die mathematisch-historische Forschung. Berl. Math. Ges. Ber. 1, p. 17-19 (1902) (JFM 33.0051.03)

[10] Felix Müller: Zur Frage über die Abkürzungen der Titel mathematischer Zeitschriften. Bibl. Math. 3 (3), p. 235-237 (1902) (JFM 34.0045.02)

[11] Felix Müller: Zur Frage der Begründung einer mathematischen Zentralbibliothek. Bibl. Math.4 (3), p. 389-391 (1903) (JFM 34.0046.03)

[12] Felix Müller: Gedenktagebuch für Mathematiker. Leipzig: G.B.G. Teubner. 121 p. (1912) (JFM 43.0001.03)

[13] Felix Müller: Versuch einer Gruppierung der neueren mathematisch-historischen Schriften (1887-1911). Zs. f. math. u. naturw. Unterr. 44, p. 461-463 (1913) (JFM 44.0043.02)
Ausgewählte Artikel über das Jahrbuch

[14] Audin, Michèle: La guerre des recensions. Autour d'une note d'André Weil (1940.) Math. Semesterber. 59, No. 2, p. 243-26o (2012) (Zbl1317.01006)

[15] Hans Aldebert; Heinz Bauer; Jean Boclé: Christian Pauc - a French mathematician as a pioneer of German-French friendship. (Christian Pauc - ein französischer Mathematiker als Wegbereiter der deutsch-französischen Freundschaft.) Mitt. Dtsch. Math.-Ver. 3, No. 2, p. 24-26 (1995) (Zbl1288.01028)

[16] Heinrich Begehr et al.: Mathematik in Berlin. Band 1, Band 2. Aachen: Shaker Verlag, 668 p. (1998) (Zbl1155.00322, Zbl1155. o0323)

[17] Leo Corry: From Algebra (1895) to Modern Algebra (1930): changing conceptions of a discipline - a guided tour using the Jahrbuch über die Fortschritte der Mathematik. p. 221-243 (2007) (Zbl1136.01006)

[18] Gabriele Dörflinger: Felix Müller; das Jahrbuch über die Fortschritte der Mathematik. Heidelberger Texte zur Mathematikgeschichte. (2013)

[19] Christophe Eckes: Organiser le recrutement de recenseurs français pour le Zentralblatt à l'automne 1940: les premiers liens entre Harald Geppert, Helmut Hasse et Gaston Julia sous l'occupation.. Revue d'Histoire des Mathématiques Rev. Hist. Math. 24, No. 2, p. 259-329 (2018) (Zblo7062737)

[20] Hans Becker, Bernd Wegner: ERAM - Digitisation of Classical Mathematical Publications. in Research and Advanced Technolgy for Digital Libraries, 4th Conference ECDL 2000, p. 424-427.

[21] Silke Göbel, Bernd Wegner: Das Zentralblatt als Zugang zur mathematischen Literatur von 1868 bis heute. Mitt. Dtsch. Math.Ver. 12 (1), p. 48-49 (2004) (Zbl1126.00312)

[22] Silke Göbel: Jahrbuch über die Fortschritte der Mathematik and Zentralblatt MATH - Reporting on more than 140 years of mathematics. Eur. Math. Soc. Newsl. 73, p. 45-46 (2009) (Zbl1189.01015)

[23] Silke Göbel: 8o years of Zentralblatt für Mathematik. Glimpses into the history of Zentralblatt für Mathematik. (8o-jähriges Jubiläum des Zentralblatt für Mathematik. Einblicke in die Geschichte des Zentralblattes.) Mitt. Dtsch. Math.-Ver. 19, No. 4, 223-227 (2011) (Zbl1235.01037)

[24] Eugen Jahnke: Nachruf auf Emil Lampe. Arch. der Math. u. Phys. (3) 28 (1919-20), p. 1-16 (1919) (JFM 47.0865.04)

[25] Walter Ledermann, Peter M. Neumann: The life of Issai Schur through letters and other documents, in Joseph, Anthony et al., Studies in memory of Issai Schur, p. xlv-xc (2003) (Zblio41. 01010)

[26] G. A. Miller: Some thoughts on modern mathematical research. Science 35, p. 877-887 (2012) (JFM 43.0088.03)

[27] G. A. Miller: Group theory reviews in the Jahrbuch über die Fortschritte der Mathematik, Bull. Am. Math. Soc. 27, 459-462 (1921) (JFM 48.0041.04)

[28] Eduardo L. Ortiz, Allan Pinkus, Herman Müntz: a mathematician's odyssey. Math. Intell., 27, No.1, p. 22-31, (2005) (Zbl1154.01326)

[29] Maximilian Pinl: Feigl zum Gedächtnis. Jahresber. Dtsch. Math.Ver.7o, p. 53-6o (1967) (Zblo15624702)

[30] Reinhard Siegmund-Schultze: Mathematische Berichterstattung in Hitlerdeutschland - Der Niedergang des Jahrbuches über die Fortschritte der Mathematik. Göttingen: Vandenhoeck \& Ruprecht. (1993), 263 p. (Zblo795.01015)

[31] Reinhard Siegmund-Schultze, The failed fusion of two mathematical reviewing journals 1932-1934 - An episode from the history of Dutch-German mathematical relations. CWI Q.9, No. 1-2, p. 131-135, 1996 (Zblo880.01015)

[32] Wolfram Sperber: Mathematical research data and information services, in Greuel, Gert-Martin (ed.) et al., Mathematical software - ICMS 2016. $5^{\text {th }}$ international conference, Berlin, Germany, July 11-14, 2016. Proceedings. Cham: Springer. Lecture Notes in Computer Science 9725, p. 429-433 (2016) (Zblo6630689) 
[33] Roland Wagner-Döbler, Jan Berg: Nineteenth-century mathematics in the mirror of its literature: A quantitative approach. Hist. Math. 23, No. 3, p. 288-318 (1996) (Zblo861.01019)

\section{Weitere Internetressourcen}

[34] Bullettino di bibliografia e di storia delle scienze matematiche e fisiche, archive.org/stream/bullettinodibibl2oroma/ bullettinodibibl2oroma_djvu.txt

[35] Bulletin des sciences mathématiques et astronomique, www.numdam.org/journals/BSMA/

[36] Jahrbuch Datenbank, www.emis.de/MATH/JFM/

[37] planck.bbaw.de/onlinetexte/Max-Henoch-Stiftung_Statut.pdf

[38] planck.bbaw.de/onlinetexte/Nachweise_zum_Wirken_Max_ Planck.pdf

[39] planck.bbaw.de/planckiana/downloads/II-VII-42_1r.pdf

[40] planck.bbaw.de/planckiana/downloads/II-VII-42_1v.pdf

[41] planck.bbaw.de/planckiana/downloads/II-VII-42_58.pdf

[42] Répertoire bibliographique des sciences mathématiques, fr.wikipedia.org/wiki/Répertoire_bibliographique_des_ sciences_mathématiques

[43] Revue semestrielle des publications mathématiques, archive.org/details/revuesemestrielo2unkngoog/

[44] WDML, www.mathunion.org/ceic/library/world-digitalmathematics-library-wdml

[45] zbMATH, www.zbmath.org

\section{Bildnachweise}

S. 97:

[Felix Müller] Felix Müller, Gedenktagebuch für Mathematiker. 3. Aufl., Teubner, Leipzig 1912

[Albert Wangerin] Virtuelles Museum des Instituts für Mathematik der Martin-Luther-Universtät Halle-Wittenberg zur Geschichte der Mathematik in Wittenberg und Halle,

disk.mathematik.uni-halle.de/history/wangerin

[Emil Lampe] Rudolf Dührkoop, Die Königliche Technische Hochschule zu Berlin, Kamera-Bildnisse. Berlin 1907

S. 98 :

[Max Henoch] Foto: F. Jacobeit. Aus einem Album der Mathematischen Gesellschaft Hamburg. Oberwolfach Photo Collection, g Mathematisches Forschungsinstitut Oberwolfach

[Arthur Korn] Pressestelle der TU Berlin,

Kopie aus dem Bundesarchiv Koblenz

[Leon Lichtenstein] Professorenkatalog der Universität Leipzig, www.mathematik.uni-leipzig.de/preprints/p1502.0020.pdf, Wikimedia Commons, commons.wikimedia.org/wiki/File:Leon_ Lichtenstein.png

S. 99

[Georg Feigl] Stefan Fabel, Geschichte der Mathematik auf einer CD-ROM, Facharbeit LK Mathematik, Miller Gymnasium 1997/99 sfabel.tripod.com/mathematik/images/Feigl.jpeg

[Hans Freudenthal] Foto: Gerd Fischer, 1984. Oberwolfach Photo

Collection, Mathematisches Forschungsinstitut Oberwolfach

S. 101

[Helmut Grunsky] Würzburger Ausstellungen zur Mathematik und ihrer Geschichte, Ausstellung zum 100. Geburtstag von Prof. Dr. Helmut Grunsky 11.7.1904-5. 6.1986,

www.history.didaktik.mathematik.uni-wuerzburg.de/ausstell/ grunsky/Bild $7 \cdot$ html

[Christian Pauc] H. Aldebert, H. Bauer und J. Boclé,

Christian Pauc - Ein französischer Mathematiker als Wegbereiter der deutsch-französischen Freundschaft. Mitteilungen der Deutschen Mathematiker-Vereinigung 3 (1995), S. 24-26

Doi 10.1515/dmvm-1995-0211

[Hermann Ludwig Schmid] Berliner Mathematische Gesellschaft, Privatbesitz von P. Roquette

S. 104

[Bernd Wegner] Mathematik im Blickpunkt. FIZ Karlsruhe, 2008

[Keith Dennis] www.ams.org/images/Dennis.jpg

\author{
Dr. Silke Göbel \\ Am Großen Wannsee 49a, 14109 Berlin \\ silke.goebel@berlin.de \\ Dr. Wolfram Sperber \\ Doßstraße 6b, 12621 Berlin \\ w_sperber@t-online.de \\ Prof. Dr. Dr.h.c. Bernd Wegner \\ zblwegner@googlemail.com
}

Silke Göbel war von 1999 bis 2011 Mitarbeiterin in der Redaktion des Zentralblattes zbMATH. Sie studierte an der FU Berlin Mathematik und an der Humboldt-Universität zu Berlin Information und Dokumentation. Ihre Doktorarbeit verfasste sie über Strukturen in reellen

Banachräumen. Später erweiterte sie ihre Interessen auf die Geschichte der Mathematik und Informationswissenschaft. Sie war federführende Mitarbeiterin im Projekt Digitalisierung des Jahrbuches über die Fortschritte der Mathematik (Electronic Research Archive of Mathematics [ERAM]).

Wolfram Sperber war nach dem Mathematik-Studium in Leipzig (1971-1975) wissenschaftlicher Mitarbeiter von 1975-1985 an der IH Cottbus (heute BTU Cottbus-Senftenberg); 1981 Promotion zu Problemen der optimalen Steuerung an der Bergakademie Freiberg; 1985-1991 wissenschaftlicher Mitarbeiter am Karl-Weierstrass-Institut der Akademie der DDR (numerische Lösung partieller DGl.); ab 1992 Arbeit in Projekten zur elektronischen Information und Kommunikation in der Mathematik, u. a. im Jahrbuch-Projekt und im Math-Net-Projekt am ZIB Berlin, der SUB Göttingen und der TU Berlin; 2006-2018 Fachredakteur und Projektarbeit beim FIZ Karlsruhe, Zentralblatt für Mathematik, u. a. im swMATH Projekt; seit 2018 im Ruhestand.

Bernd Wegner war ab 1972 Professor für Mathematik an der TU Berlin. Chefredakteur beim Zentralblatt für Mathematik von 1974 bis 2012; einige Zeit Chefredakteur von MathEduc; Leitung von verschiedenen Projekten der DFG und ausländischer Partner sowie von Projekten im EFP, ERAM, EMANI, RusDMl u.v. m. 